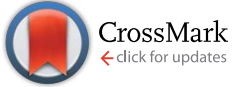

Cite this: RSC Adv., 2017, 7, 1932
Received 22nd November 2016 Accepted 21st December 2016

DOI: 10.1039/c6ra27158h

www.rsc.org/advances

\section{Enhancement of the helical twisting power with increasing the terminal chain length of nonchiral bent-core molecules doped in a chiral nematic liquid crystal}

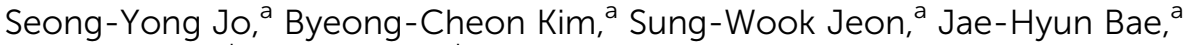 \\ Martin Walker, ${ }^{b}$ Mark Wilson, ${ }^{\text {b Suk-Won Choi }}{ }^{* a}$ and Hideo Takezoe*c \\ In this work, we prepared a series of nonchiral bent-core molecules with different terminal alkoxy chain \\ lengths, and measured the helical twisting power (HTP) of the bent-core molecules doped in a chiral \\ nematic liquid crystal $(\mathrm{N} * \mathrm{LC})$. We investigated the doping effect through the colour change and spectral \\ change due to the Bragg (selective) reflection and found that the bent-core molecules with longer \\ alkoxy chains showed stronger HTP under chiral circumstances such as N*LC. Namely, not only the axial \\ conformations at wings linked to the bent central unit but also the length of the alkoxy chains at the \\ terminal positions of the bent-core molecules play an important role in the resulting unusual chiral \\ behaviour. A preliminary stochastic dynamics simulation to determine the distribution of the chirality \\ order parameters was made, being consistent with the present experimental result.
}

\section{Introduction}

A chiral nematic liquid crystal $\left(\mathrm{N}^{*} \mathrm{LC}\right)$ phase possessing a helical structure can be readily produced by mixing a nonchiral nematic liquid crystal (NLC) with a small amount of chiral molecules (a chiral dopant). The helical structure of the $\mathrm{N}^{*} \mathrm{LC}$ is indicative of the chirality of the system and the pitch is governed by the amount and chiral strength of the chiral dopant. The helical pitch decreases with increasing chiral dopant content. As such, the inverse of the helical pitch is proportional to the mixing rate of the chiral dopant. When plotted in a graph, the slope of the inverse of the helical pitch against the mixing rate of the chiral dopant defines the chiral strength, i.e., the helical twisting power (HTP), of the mixed chiral dopant. A steeper slope indicates stronger HTP, while a gentle one denotes weaker HTP. Conversely, the mixing of nonchiral molecules elongates the helical pitch of the N*LC, which occurs because of the "diluted" chirality resulted from the addition of nonchiral molecules. ${ }^{1}$

Intriguingly, the idea discussed above is no longer applicable to nonchiral bent-core molecular systems. Nonchiral bent-core molecules sometimes behave as chiral ones, and doping

${ }^{a}$ Department of Advanced Materials Engineering for Information and Electronics (BK21Plus), Kyung Hee University, Yongin-shi, Gyeonggi-do, 446-701, Korea. E-mail: schoi@khu.ac.kr

${ }^{b}$ Department of Chemistry, Durham University, Lower Mountjoy, South Road, DH1 $3 L E, U K$

'Toyota Physical and Chemical Research Institute, 41-1 Yokomichi, Nagakute, Aichi, 480-1192, Japan. E-mail: htakezoe@yf6.so-net.ne.jp
$\mathrm{N}^{*}$ LCs with nonchiral bent-core molecules reduces the helical pitch of the $\mathrm{N}^{*} \mathrm{LCs}$, or strengthens the chirality of the $\mathrm{N}^{*} \mathrm{LCs} .{ }^{2}$ This behaviour was qualitatively explained as follows. Bent-core molecules are nonchiral but have two axially chiral conformers; ${ }^{1}$ when such molecules are dissolved in a chiral environment such as a N*LC, one of the chiral conformers is predominantly stabilized. Therefore, the chirality of the $\mathrm{N}^{*} \mathrm{LC}$ increases, resulting in the reduction of the helical pitch. ${ }^{2} \mathrm{~A}$ similar phenomenon was also observed in the chiral smectic $\mathrm{C}(\mathrm{Sm} * \mathrm{C}$ and $\mathrm{Sm}^{*} \mathrm{C}_{\mathrm{A}}$ ) mixed with bent-core molecules ${ }^{3}$ and rod-shaped molecules with an ester linkage. ${ }^{4}$ Thus, the axially chiral conformation of such nonchiral molecules plays an important role in the stated unusual phenomena. Herein, we performed pitch measurements in an N*LC compound mixed with nonchiral bent-core homologues possessing a central bent-core phenyl unit with different terminal alkoxy chain lengths. The HTP of the doped bent-core molecule in the $\mathrm{N}^{*} \mathrm{LC}$ strongly depended on the terminal alkoxy chain length.

\section{Experimental}

\section{Materials}

Fig. 1 illustrates the chemical structures of the molecules used: a host chiral compound $\mathbf{A}$ purchased from 4 Chem Laboratory and three bent-core homologues BC- $\boldsymbol{m}$ with different terminal alkoxy chain lengths ( $m=8,12$, and 16$)$, which were synthesized due to a previous report. ${ }^{5}$ The host compound $\mathbf{A}$, which was employed by Thisayukta et al., ${ }^{2}$ possesses the N*LC phase over a wide temperature range. One of the nonchiral bent-core 


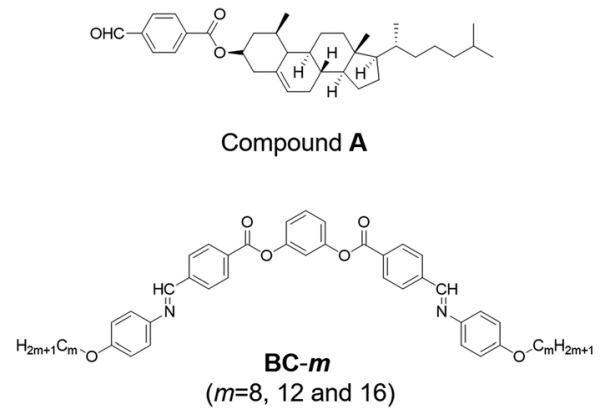

Fig. 1 Chemical structures of compounds A and BC- $m$, where $m=8$, 12 , and 16

molecules (BC-12) was also used by Thisayukta et al., ${ }^{2}$ although they did not use its homologues. In BC-m, an ester group links a phenyl bent central unit to rod-like wings. We previously reported that these ester linkages play a key role in the chiral behaviour of nonchiral bent-core molecular systems based on vibrational circular dichroism measurements. ${ }^{6}$

\section{Methods}

We doped compound A with the bent-core homologues, and then investigated the doping effect by monitoring the colour and spectral change due to the Bragg (selective) reflection change. We prepared sample mixtures of chiral compound $\mathbf{A}$ (host) with nonchiral BC-m (guest) in various weight fractions of up to $65: 35$. Pure compound $\mathbf{A}$ and mixture samples were sandwiched between two glass plates. The cells (sample thickness: $\sim 4 \mu \mathrm{m}$ ) were cooled to a solid state and heated until they reached the N*LC phase. The planar alignment of the cells was confirmed by using a polarizing optical microscope (POM) equipped with a temperature control unit (Mettler FP-82). The selective reflection spectra in the $\mathrm{N}^{*} \mathrm{LC}$ phase were obtained by a multichannel spectrometer (USB-2000, Ocean Optics). The optical pitch $n P$ was determined from the central wavelength of the reflection band. Here, $n$ is the average refractive index and $P$ is the helical pitch of the mixture. By assuming $n=1.5$ (ref. 7) irrespective of $m$ and the doping content, we obtained $P$ and used to determine HTP.

\section{Results and discussion}

First, the phenomenon reported by Thisayukta et $a .^{2}$ was reproduced using the mixtures of compound A and BC-8. Fig. 2 illustrates the planar textures in the $\mathrm{N}^{*} \mathrm{LC}$ phase of pure compound $\mathbf{A}$, and in the mixtures with $15 \mathrm{wt} \%$ and $30 \mathrm{wt} \%$ of BC-8. Here, the textures were observed at the same reduced temperature $T-T_{\mathrm{c}}=20{ }^{\circ} \mathrm{C}$, where $T_{\mathrm{c}}$ is the transition temperature from the crystal to the $\mathrm{N}^{*} \mathrm{LC}$ phase on heating. Reflectance spectra are also shown in Fig. 2. When the concentration of BC-8 was increased from $0 \mathrm{wt} \%$ to $30 \mathrm{wt} \%$, the reflection colour clearly changes from orange to green, implying tightening of the helical pitch. This is the unusual behaviour observed when doping $\mathrm{N}^{*} \mathrm{LCs}$ with nonchiral molecules.
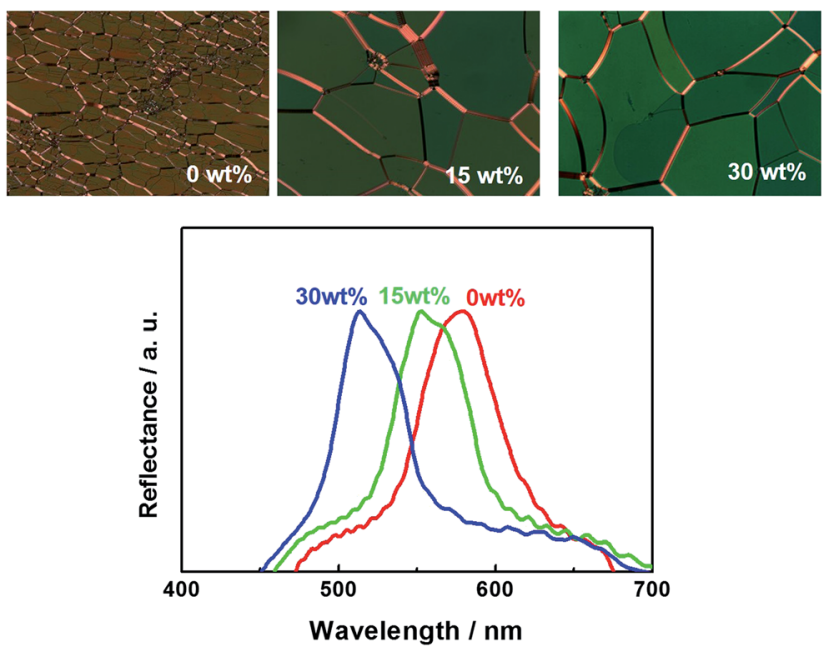

Fig. 2 Planar textures in the $\mathrm{N} * \mathrm{LC}$ phase of pure compound $\mathrm{A}$, and in the mixtures containing $15 \mathrm{wt} \%$ and $30 \mathrm{wt} \%$ of BC-8. Reflectance spectra are also shown.

Fig. 3 shows the planar textures in the $\mathrm{N}^{*} \mathrm{LC}$ phase of the mixtures of $75 \mathrm{wt} \%$-compound A with $25 \mathrm{wt} \%$ of BC-8, BC-12, and $\mathbf{B C - 1 6}$ at the same reduced temperature $\left(T-T_{\mathrm{c}}=20^{\circ} \mathrm{C}\right)$. Reflectance spectra are also shown in Fig. 3, demonstrating the reflection colour shift to shorter wavelengths in the mixtures doped with bent-core molecules containing longer terminal alkoxy chain lengths.

The inverse of the pitch $(1 / P)$ at the reduced temperature $T-$ $T_{\mathrm{c}}=20{ }^{\circ} \mathrm{C}$ is plotted against the content (mol\%) of BC- $\boldsymbol{m}(m=8$, 12, and 16) in Fig. 4(a). The slope of each solid line defines the HTP of each doped bent-core molecule. The slope is steeper for BC-m, which has a longer terminal alkoxy chain. Hence, the compounds with longer terminal chains show stronger HTP, as shown in Fig. 4(b). The estimated HTP values of BC-8, BC-12 and
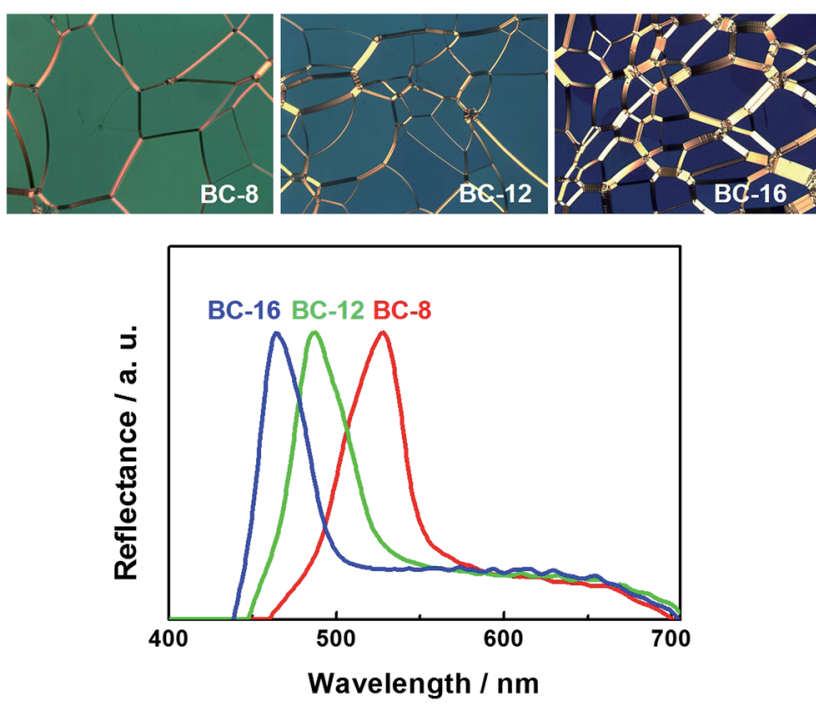

Fig. 3 Planar textures in the $\mathrm{N} * \mathrm{LC}$ phase of the mixtures containing 75 wt $\%$ of compound A with 25 wt\% of BC-8, BC-12, and BC-16. Reflectance spectra are also shown. 

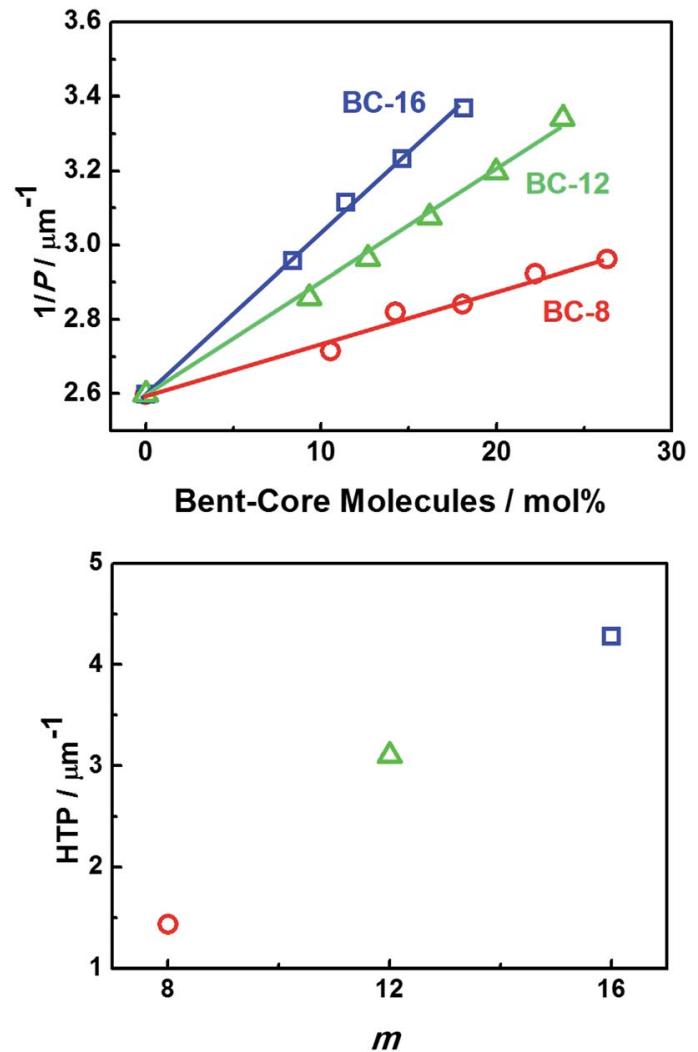

Fig. 4 (a) Reciprocal pitch (1/P) against the BC- $m(m=8,12$, and 16) content (mol\%). (b) HTP vs. $m$.

BC-16 were, respectively, 1.4, 3.1, and $4.3 \mu \mathrm{m}^{-1}$. Note that the HTP values of nonchiral bent-core molecules are relatively small compared with those of commercial chiral dopant molecules, ${ }^{8}$ although the host molecule used here is a cholesterol derivative, being different from nonchiral calamitic host molecules used in conventional HTP measurements.

Chiral molecules with high HTP are useful as dopants to induce chiral phases such as $\mathrm{N}^{*}, \mathrm{SmC}^{*}$, and blue phases (BP) with small contents. Hence, the syntheses and development of chiral molecules with a high HTP have been extensively made. Previous works on HTP reported the effect of chiral molecules with different chiral structures such as substituent groups on binaphthyl derivatives, ${ }^{9}$ the number of chiral groups, ${ }^{\mathbf{1 0 , 1 1}}$ and the end chain length. ${ }^{12-15}$ It is known that the chiral structure strongly affects the HTP, whereas the end chain length does not. In fact, different chiral structures induce several-times different HTP. ${ }^{9-11}$ However, the HTP varies only by a few tens of percent when the end chain length is changed. ${ }^{\mathbf{1 0 , 1 3 , 1 4}}$ The only exception is a sugar-appended Schiff base chiral rod-coil amphiphile with different alkoxy end chains, in which the HTP changes by several times and even shows the highest HTP in a compound with a medium alkoxy chain length $(m=13$ among $m=9-22) .{ }^{12}$

As mentioned above, the results of this study (Fig. 4) indicate that the HTP depends strongly on the terminal alkoxy chain length of the bent-core molecule; HTP enhanced by about 3 times from $m=8$ to $m=16$. HTP drastically increases with increasing end-chain length. This means that compounds with longer end chains possess a stronger chirality and/or transfer their chirality to the $\mathrm{N}^{*} \mathrm{LC}$ more efficiently under chiral conditions. The higher conformational flexibility may be the underlying reasoning, but the full understanding requires further studies. A preliminary stochastic dynamics simulation for $n=8$ and 12 was carried out to determine the distribution of the chirality order parameters of these compounds, as reported by Earl et al. ${ }^{\mathbf{1 6 , 1 7}}$ The histograms obtained show a wide distribution, which means that there are a variety of chiral conformations of these molecules. Overall chirality is essentially zero, since the distribution is symmetric. However, if the chiral balance is slightly distorted under a chiral circumstance, a large HTP is possibly obtained. In the present preliminary simulation, the distribution for the compound with $n=12$ is wider than that for the compound with $n=8$. This result is consistent with the present experimental result. The full details will be reported soon in a separate paper.

\section{Conclusions}

In summary, a series of nonchiral bent-core molecules with different terminal alkoxy chain lengths were prepared and doped into a host chiral compound possessing the $\mathrm{N}^{*}$ phase. We investigated the doping effect through the colour change and spectral change due to the Bragg (selective) reflection. We found that the pitch of the $\mathrm{N}^{*} \mathrm{LCs}$ became remarkably shorter when doped with molecules possessing longer terminal alkoxy chains. Bent-core molecules with longer alkoxy chains showed stronger HTP under chiral conditions such as $N^{*} \mathrm{LC}$. The result is consistent with a preliminary stochastic dynamics simulation for chirality order parameter of the compounds used.

\section{Acknowledgements}

This work was supported by the Basic Science Research Program (NRF-2016R1D1A1A09917580) through the National Research Foundation of Korea (NRF). We acknowledge Prof. K. Kishikawa of Chiba University and Prof. K. Akagi of Kyoto University for useful information.

\section{Notes and references}

1 H. Takezoe, Top. Curr. Chem., 2012, 318, 303.

2 J. Thisayukta, H. Niwano, H. Takezoe and J. Watanabe, J. Am. Chem. Soc., 2002, 124, 3354.

3 E. Gorecka, M. Cepic, J. Mieczkowski, M. Nakata, H. Takezoe and B. Zeks, Phys. Rev. E: Stat., Nonlinear, Soft Matter Phys., 2003, 67, 061794.

4 S.-W. Choi, K. Fukuda, S. Nakahara, Y. Takanishi, K. Ishikawa, J. Watanabe and H. Takezoe, Chem. Lett., 2006, 35, 896.

5 T. Akutagawa, Y. Matsunaga and K. Yasuhara, Liq. Cryst., 1994, 17, 659.

6 S.-W. Choi, S. Kawauchi, S. Tanaka, J. Watanabe and H. Takezoe, Chem. Lett., 2007, 36, 1018.

7 T. Tako, T. Akahane and S. Masubuchi, Jpn. J. Appl. Phys., 1975, 14, 425. 
8 H.-C. Jeong, S. Aya, S. Kang, F. Araoka, K. Ishikawa and H. Takezoe, Liq. Cryst., 2013, 40, 951; K. Kim, S. Kim, S.-Y. Jo and S.-W. Choi, J. Inf. Disp., 2015, 16, 155.

9 M. Goh and K. Akagi, Liq. Cryst., 2008, 35, 953.

10 K. Fukuda, H. Suzuki, J. Ni, M. Tokita and J. Watanabe, Jpn. J. Appl. Phys., 2007, 46, 5208.

11 K. Fukuda, S. Edo, M. Muto, M. Tokita and J. Watanabe, Jpn. J. Appl. Phys., 2008, 47, 8479.

12 T.-F. Lin, R.-M. Ho, C.-H. Sung and C.-S. Hsu, Chem. Mater., 2008, 20, 1404.
13 W. Huang, X. G. Zhang, G. D. Yang, Z. Y. Bian, X. J. Wu, G. J. Ma, Q. Huang and H. Yang, Chin. Chem. Lett., 2009, 20, 1435.

14 K. Kishikawa, T. Sugiyama, T. Watanabe, S. Aoyagi, M. Kohri, T. Taniguchi, M. Takahashi and S. Kohmoto, J. Phys. Chem. B, 2014, 118, 10319.

15 D. Fu, J. Li, J. Wei and J. Guo, Soft Matter, 2015, 11, 3034. 16 D. J. Earl and M. R. Wilson, J. Chem. Phys., 2003, 119, 10280. 17 D. J. Earl, M. A. Osipov, H. Takezoe, Y. Takanishi and M. R. Wilson, Phys. Rev. E: Stat., Nonlinear, Soft Matter Phys., 2005, 71, 021706. 